\title{
Open versus minimal invasive radical cystectomy
}

\author{
Danielle C. van Diepen, Tahlita C. M. Zuiverloon, Sjoerd O. Klaver, Joost L. Boormans \\ Department of Urology, Erasmus MC Cancer Institute, Rotterdam, The Netherlands \\ Correspondence to: Joost L. Boormans. Associate professor of Urology, Department of Urology, Erasmus MC Cancer Institute, Dr Molewaterplein 40, \\ 3015 GD, Rotterdam, The Netherlands. Email: j.boormans@erasmusmc.nl. \\ Comment on: Khan MS, Omar K, Ahmed K, et al. Long-term Oncological Outcomes from an Early Phase Randomised Controlled Three-arm Trial \\ of Open, Robotic, and Laparoscopic Radical Cystectomy (CORAL). Eur Urol 2020;77:110-8.
}

Submitted Apr 20, 2020. Accepted for publication May 29, 2020.

doi: $10.21037 /$ tau-20-878

View this article at: http://dx.doi.org/10.21037/tau-20-878

With great interest we read the report by Khan et al. and commend the authors on having conducted the CORAL trial, which is one of three currently published randomized controlled trials (RCTs) reporting on long-term oncological outcomes of open (ORC) versus minimal invasive radical cystectomy (MIRC) for muscle-invasive bladder cancer (MIBC) or high-risk non muscle-invasive bladder cancer (HRNMIBC) (1-3).

Although the main incentive for the introduction of MIRC has always been to reduce perioperative morbidity and length of hospital stay, the introduction of a new surgical technique is only justified if the oncological outcomes are equal or better than the current gold standard, which is ORC. Following their initial publication on perioperative morbidity, Khan et al. recently reported the long-term oncological outcomes from their single institution randomized controlled trial (RCT) of ORC versus laparoscopic (LRC) and robot-assisted radical cystectomy (RARC) with extracorporeal urinary diversion in a total of 59 MIBC $(\mathrm{N}=38)$ or HRNMIBC $(\mathrm{N}=21)$ patients $(1,4)$. The clinical outcome of the patients did not differ between the treatment groups as no statistically significant differences were observed in the 5 -year recurrence-free (RFS), cancer-specific (CSS), and overall survival (OS). The 5 -year RFS was $60 \%, 58 \%$, and $71 \%$; the 5 -year CSS was $64 \%, 68 \%$, and $69 \%$; and the 5 -year OS was $55 \%, 65 \%$, and $61 \%$ for ORC, RARC, and LRC, respectively. These findings are in line with the results of two other RCTs by Bochner et al. and Venkatramani et al. $(2,3)$ that randomized patients between ORC and RARC. In addition, the 5-year CSS rate observed in the CORAL trial was equivalent to the 5 -year CSS of $66.8 \%$ reported by Hautmann et al. in a cohort of 1,100 patients treated by ORC (5). These survival data support the evidence that MIRC is comparable to open surgery in terms of oncological outcomes.

The authors should be commended on their study design with well-matched study arms, adequate patient followup, and their efforts to prevent surgeon bias. Although an RCT represents a high level of evidence, even RCTs might be influenced by several biases and the CORAL trial is not exempt. Important limitations of the study are the small sample size, the single center set-up, and failure to deliver treatment to the allocated randomized treatment arms. In the ORC arm, all 20 patients underwent the allocated treatment whereas in the RARC group one patient underwent conversion to ORC and in the LRC group 5 patients (25\%) did not receive LRC; three patients were converted to RARC, one to ORC and one patient underwent chemoradiation instead of surgery. Additionally, the CORAL trial was powered to detect a difference in perioperative complication rates between the studies arms and not to test differences in survival outcomes. Moreover, because of recruitment difficulties, the trial was terminated early after inclusion of 60 instead of the aimed 141 patients. Therefore, reported results should be interpreted with great caution.

The proportion of patients who received neoadjuvant chemotherapy in the CORAL trial was small $(\mathrm{N}=6)$ but equally distributed between the study arms in three, two and one patient for ORC, RARC and LRC respectively. The clinical lymph node status was not reported and the indication for preoperative chemotherapy, either being neoadjuvant for clinically node-negative patients or induction setting for clinically node-positive patients, 
was not stated. This could possibly have had an impact on oncological survival outcomes. Moreover, the proportion of preoperative MIBC diagnosis seemed overrepresented in the LRC group; $74 \%$ versus $60 \%$ in both ORC and RARC groups.

With respect to the oncological safety of MIRC vs. ORC, two important points of discussion have been mentioned over recent years. First, it was suggested that lymph node dissections were less adequate and resulted in a lower lymph node yield for minimally invasive techniques. Secondly, concerns were raised that the pneumoperitoneum during laparoscopic surgery could enhance local tumor spread or implantation of tumor cells into the peritoneal cavity $(2,6)$. However, like the RAZOR trial, Khan et al. did not find a difference in lymph node yield and local recurrence rate between the different surgical modalities $(1,3)$.

Lastly, both patient and surgeon bias were also noted in the CORAL trial. Of the 164 patients eligible for inclusion, $35 \%$ declined to participate because of patient preferences for a specific surgical approach. The authors tried to minimize surgeon bias by ensuring all surgeons completed their learning curve with more than 110 cystectomies performed per surgeon for the respective modalities but evidence is lacking on what the optimal number of procedures performed should be for a surgeon who completed the learning curve. Therefore, differences in surgical skills could potentially have influenced the results of the CORAL trial.

Current literature suggests that oncological outcomes for MIBC are predominantly determined by tumor biology and that the surgical modality is unlikely to play a significant role in the risk of disease recurrence after cystectomy. Therefore, the advantage of MIRC over ORC should lay in less perioperative morbidity because cystectomy itself is a challenging surgical procedure with high morbidity rates. In addition, reduction of perioperative morbidity lowers additional costs of auxiliary diagnostic and therapeutic procedures for peri- and postoperative complications. Thus far, in all RCTs that compared ORC versus MIRC the urinary diversion was done extracorporeal. However, since morbidity from a cystectomy is mainly caused by the reconstructive phase for which parts of the small intestine are used, extracorporeal urinary diversions for which a laparotomy is mandatory could dilute the potential advantage of MIRC and open reconstruction in terms of perioperative morbidity. We therefore eagerly await results of the iROC study, a multicenter prospective RCT comparing RARC with intracorporeal urinary diversion versus ORC (7). Furthermore, minimally invasive techniques provide an important advantage because the pneumoperitoneum and superior visibility of small blood vessels lead to reduced perioperative blood loss and the need for blood transfusions. Also, the median length of hospital stay is shorter for MIRC than for ORC, while ORC shows a reduced operating time and lower total costs.

Taken together, no differences have been observed between MIRC and ORC in terms of complication rate, pathological stage, lymph node yield and positive surgical margins. Now multiple RCTs, including the CORAL trial, show that long-term oncological outcomes are equivalent for MIRC and ORC and that perioperative morbidity is favorable for MIRC. Are these benefits of MIRC, as part of a larger context of complete patient recovery over time, sufficient to change current clinical practice? The surgical technique is one aspect of the complete treatment of MIBC and up to now no solid evidence is provided that minimal invasive techniques strongly outweigh the gold standard ORC in terms of costs, perioperative and longterm oncological outcome. Furthermore, patient and tumor characteristics should not be neglected and surgeon and hospital volume are well-established key determinants of outcome for both techniques. By developing minimally invasive techniques, we have broadened our surgical repertoire and are now able to offer patients a more personalized treatment option. Hence, for us the challenge does not lie in identifying the superiority of one surgical technique over the other, but rather in the imperative question of which patients to select for either technique.

\section{Acknowledgments}

Funding: None.

\section{Footnote}

Provenance and Peer Review: This article was commissioned by the editorial office, Translational Andrology and Urology. The article did not undergo external peer review.

Conflicts of Interest: All authors have completed the ICMJE uniform disclosure form (available at http://dx.doi. org/10.21037/tau-20-878). The authors have no conflicts of interest to declare.

Ethical Statement: The authors are accountable for all aspects of the work in ensuring that questions related 
to the accuracy or integrity of any part of the work are appropriately investigated and resolved.

Open Access Statement: This is an Open Access article distributed in accordance with the Creative Commons Attribution-NonCommercial-NoDerivs 4.0 International License (CC BY-NC-ND 4.0), which permits the noncommercial replication and distribution of the article with the strict proviso that no changes or edits are made and the original work is properly cited (including links to both the formal publication through the relevant DOI and the license). See: https://creativecommons.org/licenses/by-nc-nd/4.0/.

\section{References}

1. Khan MS, Omar K, Ahmed K, et al. Long-term Oncological Outcomes from an Early Phase Randomised Controlled Three-arm Trial of Open, Robotic, and Laparoscopic Radical Cystectomy (CORAL). Eur Urol 2020;77:110-8.

2. Bochner BH, Dalbagni G, Marzouk KH, et al. Randomized trial comparing open radical cystectomy and robot-assisted laparoscopic radical cystectomy: oncologic

Cite this article as: van Diepen DC, Zuiverloon TCM, Klaver SO, Boormans JL. Open versus minimal invasive radical cystectomy. Transl Androl Urol 2020;9(6):2471-2473. doi: 10.21037/tau-20-878 outcomes. Eur Urol 2018;74:465-71.

3. Venkatramani V, Reis IM, Castle EP, et al. Predictors of Recurrence, and Progression-Free and Overall Survival following Open versus Robotic Radical Cystectomy: Analysis from the RAZOR Trial with a 3-Year Followup. J Urol 2020;203:522-9.

4. Khan MS, Gan C, Ahmed K, et al. A single-centre early phase randomised controlled three-arm trial of open, robotic, and laparoscopic radical cystectomy (CORAL). Eur Urol 2016;69:613-21.

5. Hautmann RE, de Petriconi RC, Pfeiffer C, et al. Radical cystectomy for urothelial carcinoma of the bladder without neoadjuvant or adjuvant therapy: long-term results in 1100 patients. Eur Urol 2012;61:1039-47.

6. Nguyen DP, Al Hussein Al Awamlh B, Wu X, et al. Recurrence patterns after open and robot-assisted radical cystectomy for bladder cancer. Eur Urol 2015;68:399-405.

7. Catto JWF, Khetrapal P, Ambler G, et al. Robot-assisted radical cystectomy with intracorporeal urinary diversion versus open radical cystectomy (iROC): protocol for a randomised controlled trial with internal feasibility study. BMJ Open 2018;8:e020500. 